\title{
La enseñanza del género explicación secuencial en el aula de Prácticas del Lenguaje
}

Teaching of sequential explanation as a genre in Spanish classrooms

\section{Florencia Gimena Nieto}

\author{
Universidad Nacional de General Sarmiento- UNGS - Buenos Aires - Argentina
}

Resumen: En este artículo se aborda la implementación de una secuencia didáctica diseñada para $3^{\circ}$ año de escuela secundaria, dentro de la asignatura Prácticas del Lenguaje (PDL). Además, se propone una mirada crítica acerca de la enseñanza de Lengua en las aulas argentinas, al tiempo que se despliegan los principios teóricos que guiaron el diseño de una propuesta didáctica específica, basada en la enseñanza de géneros. La propuesta didáctica adoptada se basa, a su vez, en los aportes realizados por la Escuela de Sydney y la Lingüística Sistémico-Funcional (LSF), a partir de la cual se logran describir distintos géneros científicos recurrentes en el ámbito de la escuela secundaria. Asimismo, en este artículo en particular, abordaremos el trabajo con el género explicación secuencial en Ciencias Naturales, por lo que se realizará una breve exposición de su estructura esquemática y recursos lingüísticos principales, así como también los resultados de su aplicación.

Palabras clave: Lingüística Sistémico-Funcional, Propuesta didáctica, Enseñanza en secundaria, Explicación secuencial.

\begin{abstract}
This paper addresses the implementation of a pedagogic sequence designed for a third grade of secondary school in the subject called Prácticas del Lenguaje (PDL), that it's similar to Spanish class. It also proposes a critical view on the teaching of language in the Argentine classrooms, while at the same time the theoretical principles that have guided a specific pedagogic proposal based on genre pedagogy are formulated. This proposal is also based on the contributions made by the Sydney School and the Systemic Functional Linguistics (SFL), on the basis of which diverse recurrent scientific genres in the secondary school environment can be described. Furthermore, in this paper in particular, we will deal with the use of the sequential explanation genre in Natural Sciences, and thus a brief explanation of the schematic structure and the main linguistic resources will be proposed, as well as the outcomes of its pedagogical application.
\end{abstract}

Keywords: Systemic Functional Linguistics, didactic proposal, secondary school, language teaching, sequential explanation. 


\section{Introducción}

El objeto de enseñanza lengua ha sido foco de cuantiosas discusiones a lo largo de la historia curricular de la educación primaria y la secundaria argentina, así como también lo ha sido el lugar de la gramática en dicha práctica, y, más tardíamente, los enfoques adoptados en la enseñanza de la lectura y la escritura (CORTÉS, 2001; OTAÑI; GASPAR, 2001). Con la ley 26.206, sancionada en el año 2006, los diseños curriculares de las asignaturas fueron reelaborados de manera global (DIRECCIÓN GENERAL DE CULTURA Y EDUCACIÓN [DGCyE], 2006). Es así que en el caso específico del área Lengua y Literatura, la asignatura tomó un nuevo nombre, Prácticas del Lenguaje, y con ello se estableció un nuevo enfoque a partir del cual se pretendió no disgregar las partes que componen el uso del lenguaje en contexto, separándose tanto del enfoque más tradicional o estructuralista, como del enfoque comunicativo. Así, la unidad tomada es el lenguaje, entendido en términos saussurianos como aquello que engloba el sistema de la lengua y su uso.

Sin embargo, a diferencia de lo que sucedió con otras disciplinas del diseño curricular, en Prácticas del Lenguaje ( $P D L)$, si bien se delimita qué se toma por objeto de enseñanza, no se explicita claramente una metodología de trabajo ni tampoco una propuesta didáctica que modele la manera de enseñar los contenidos prescriptos. Consideramos que frente a este vacío didáctico la pregunta sobre qué enseñar en PDL y cómo enseñarlo, a pesar de ser una discusión con tanta historia y tanta reflexión a cuestas, sigue siendo un interrogante legítimo en este campo, que merece ser revisitado para mejorar la práctica docente en el nivel secundario.

De esta manera, se hace necesario mencionar que la formación en este nivel atiende a tres grandes propósitos: la formación de los jóvenes en la vida social y ciudadana, la formación para el mundo del trabajo y la formación para los estudios superiores (DGCyE, 2006). En este sentido, es preciso señalar que los textos constituyen cada una de estas prácticas sociales, por lo tanto su enseñanza tiene una importancia vital a la hora de brindarles herramientas a los alumnos para su formación en los tres ámbitos considerados. Si es un derecho de los jóvenes tener acceso a la socialización, el mundo del trabajo y los estudios superiores, es una obligación de la escuela garantizar las condiciones de enseñanza-aprendizaje para que este derecho no se vulnere y todos los jóvenes tengan las mismas oportunidades.

Esta tarea es uno de los principios más contundentes, a la vez que desafiantes, para la escuela actual. Los profesores de Lengua, ciertamente, no estamos exentos de esta responsabilidad. Por este motivo, sostenemos que la enseñanza de los textos que circulan en el ámbito de las ciencias, la vida ciudadana y el mundo del trabajo debe ser el principal objeto de enseñanza de la materia PDL. El acceso a textos y géneros sofisticados que intervienen en actividades sociales más complejas, como, por ejemplo, el lenguaje y los códigos de las ciencias, o de los espacios de toma de decisiones, es lo que determina la inclusión o exclusión de los sujetos de formar parte activa de estas prácticas sociales de mayor relevancia social. De modo que, es evidente aquí que no consideramos el aprendizaje de la lectura y la escritura como habilidades separadas de cualquier otro conocimiento, sino que, por el contrario, sabemos que el conocimiento se construye en y por el lenguaje, y que como tal, el aprendizaje del lenguaje y su organización implica el aprendizaje de estructuras que permiten acceder a todo tipo de conocimiento científico y no científico (HALLIDAY, 1993).

En este trabajo se abordará precisamente esta cuestión, dado que la propuesta didáctica se basa en una opción teórica determinada, la Lingüística Sistémico-Funcional, que considera al lenguaje como creador de significado (MOYANO, 2013), por ende, asigna un rol específico a la gramática en la enseñanza de la lectura y la escritura que escapa tanto al lugar prominente como subsidiario de los elementos gramaticales (OTAÑl; GASPAR, 2001). Como alternativa, esta perspectiva ofrece una función al metalenguaje en la que su enseñanza parte de cuáles son los recursos lingüísticos que le dan identidad a un género determinado, por lo que su enseñanza cobra 
inmediato sentido si se reflexiona sobre las elecciones lingüísticas particulares de un género en el marco de una práctica social concreta.

Ademas, luego de presentar la opción teórica que nos permite delimitar qué enseñar y cómo enseñarlo, presentaremos en detalle la propuesta didáctica adaptada en Moyano (2007; 2013). Por último presentaremos el análisis y los resultados de una secuencia didáctica implementada en $3^{\circ}$ año de PDL, en la que se trabajó el género explicación secuencial en Ciencias Naturales, en especial, su propósito social, su estructura esquemática y sus recursos gramaticales.

\section{Propuesta pedagógica}

\section{Una opción teórica}

La necesidad de hacer una elección del objeto de enseñanza tiene que ver, en verdad, con hacer una elección respecto de un marco teórico que pueda proporcionar un objeto de enseñanza adecuado a los fines pedagógicos que nos atañen. En el caso de la materia Prácticas del Lenguaje en el nivel secundario, las habilidades de lectura y escritura son una parte vital en el aprendizaje del lenguaje en contexto, ya que justamente son habilidades específicas de este objeto. Sin embargo:

"estos objetos no pueden ser pensados en el vacío: se enseña a leer y a escribir algo. $Y$ ese algo son textos. Textos entendidos como unidades semióticas que pueden observarse desde dos perspectivas: como productos, en una perspectiva sinóptica, y como procesos, en una perspectiva dinámica" (MOYANO, 2017: 50).

Según Martin (1985), los textos entendidos como productos son unidades de lenguaje que producen significados relevantes en su contexto. Mientras que, como procesos, se los concibe en un devenir, en un desarrollo propio a través del tiempo en el cual produce significado en el mismo despliegue de elecciones lingüísticas realizadas allí. En este sentido, los textos siempre son unidades lingüísticas que actualizan ese reservorio potencial de opciones del lenguaje, a lo cual Halliday llama sistema (HALLIDAY, 1981 (2002); 1992 (2003)), y, simultáneamente, éstos se encuentran relacionados con su contexto. Es importante aquí mencionar que por contexto no nos referimos a ciertas condiciones materiales que lindan al texto, sino que Halliday (1991 (2007)) considera al contexto como una construcción absolutamente teórica para explicar cómo es que el texto se vincula con el proceso social en el que aparece. Por lo tanto, un texto constituye una unidad semántica que se negocia en los intercambios sociales, y de esa manera es que se relaciona con su contexto.

La relación mencionada entre texto y contexto precisa de otro concepto que media entre ambos, esto es, la realización, pensada como una especie de "encodificación" de significados (MARTIN; ROSE, 2008). Es decir, que los significados disponibles en el contexto se traducen o manifiestan en el lenguaje. Es por esto que Martin (1992), señala que el contexto es un sistema semiótico sin medio de realización, vale decir, connotativo, mientras que el lenguaje, al ser denotativo y poseer tanto plano de contenido como de expresión, provee los medios de realización al contexto, tal como se muestra en la Figura 1.

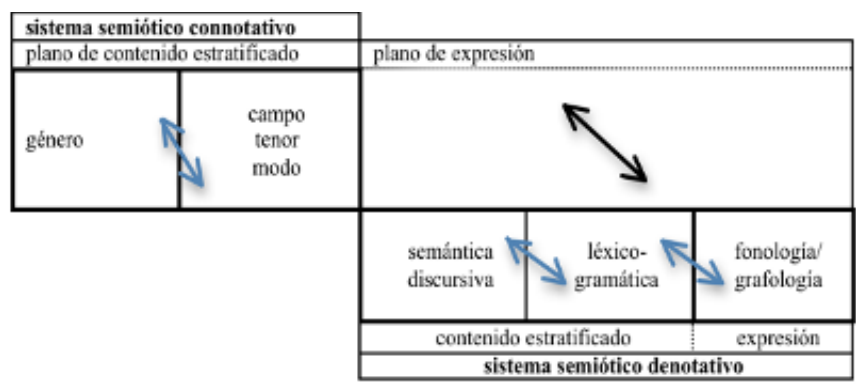

Figura 1: Estratificación de Contexto y Lenguaje (MARTIN, 1999a).

En consecuencia, esto implica que ambos sistemas semióticos, el contexto y el lenguaje, mantienen una relación de doble determinación, dado que el contexto construye el lenguaje porque los significados contextuales seleccionan las opciones del sistema de lenguaje a ser realizadas, y al mismo tiempo, la realización de elecciones lingüísticas particulares construye, a su vez, el contexto que favoreció dichas opciones para la producción de un significado determinado. Así, el contexto crea al lenguaje y el lenguaje permite la expresión de un contexto en específico. 
Asimismo, los significados del contexto y los del lenguaje se relacionan funcionalmente. Los significados del lenguaje se organizan en torno a un modelo que presenta tres metafunciones: la metafunción ideacional, interpersonal y textual.

Tal como lo explica Martin (1997) la metafunción ideacional tiene que ver con la "representación de mundo", contemplando sus procesos, los participantes que se involucran en ellos y las circunstancias en que suceden; la metafunción interpersonal tiene que ver con la interacción entre participantes, contemplando sus relaciones y las valoraciones que realizan sobre el mundo; la metafunción textual tiene que ver con el flujo de información, con cómo se organiza la información desplegada en un texto. A su vez, estas tres metafunciones se proyectan en el contexto realizando las tres variables en que se distribuyen los significados posibles: campo, tenor y modo. El campo es la variable contextual que se ocupa de las diferentes prácticas institucionalizadas en las que los humanos tomamos parte. La variable contextual de tenor se ocupa de las relaciones y los posicionamientos sociales entre sujetos. El modo, por su parte, es la variable que se ocupa del canal, por lo que involucra distintos niveles de abstracción y determina el uso de lenguaje congruente o abstracto. Así las cosas, los significados del campo se realizan a través de los recursos de la metafunción ideacional, los del tenor mediante los recursos de la metafunción interpersonal y los del modo a través de los recursos de la metafunción textual, tal como se comprueba en la Figura 2:

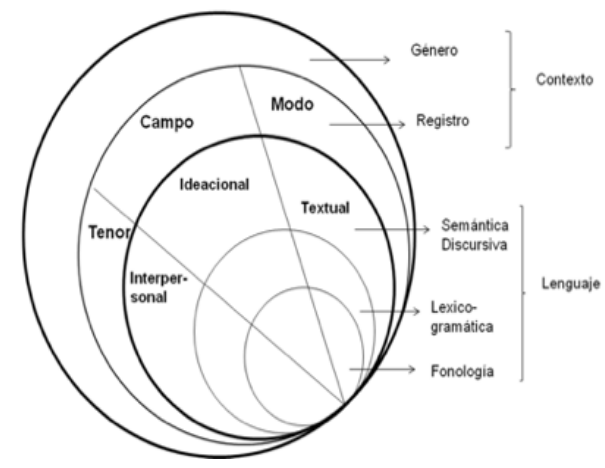

Figura 2: Contexto y Lenguaje estratificados (adaptado de MARTIN; ROSE, 2007)
En un nivel de descripción mayor, Martin (1992) propone una estratificación del contexto, que recordemos no posee plano de expresión per se, en dos niveles: registro y género. Entonces, el registro es el estrato que contiene el conjunto de variables de campo, tenor, y modo, es decir, actúa en el estrato menos abstracto del contexto, el cual simultáneamente se relaciona con el estrato semántico-discursivo del lenguaje a través de las metafunciones. En cambio, se denomina género al estrato superior del contexto, que no tiene una relación directa con las metafunciones. En este sentido, en la Figura 2, se muestra que el género se construye, por un lado, como significado, esto es, como configuración particular de campo, tenor y modo en tanto que permite la relación de estas variables y la construcción de un proceso social; y por el otro, como una serie de pasos o etapas en que los textos se estructuran, lo que se conoce teóricamente como estructura esquemática (MARTIN, 1997: 6).

Teniendo en cuenta todo lo anterior, la categoría de género se concibe como un proceso social llevado a cabo a través del lenguaje, ya sea de manera constitutiva o auxiliar de la actividad de que se trate, siempre en un ámbito específico de una cultura en particular. Como ya se mencionó, dicho proceso se lleva a cabo en pasos o etapas mediante las cuales los géneros consiguen una estructura esquemática en pos de un propósito social (MARTIN, 1997). Como los géneros son realizados a partir del lenguaje, se propone pensar que para su caracterización se deberán rastrear los recursos semántico-discursivos que predominen en el proceso social que guía la construcción del registro, y por ende, la estructura esquemática del género, con los recursos lingüísticos correspondientes.

En la conceptualización propuesta por la Lingüística Sistémico-Funcional, y específicamente a partir de Martin; Rose (2007), las instancias de un género o textos son unidades del estrato semánticodiscursivo del lenguaje, por lo cual, los significados que realicen podrán ser analizados a partir de los recursos correspondientes para ese mismo estrato, teniendo en cuenta las tres metafunciones que las atraviesan, como se graficó en la Figura 2. 
Paralelamente, el análisis de los recursos semánticodiscursivos (Cuadro 1) permite reflexionar sobre el registro de un texto, ya que los significados ideacionales se encargan de construir el campo de un texto, y los significados interpersonales habilitan la construcción del tenor y los textuales, el modo. En este sentido, el registro permite realizar el género como proceso social orientado a un fin determinado. De igual manera, el análisis desde el enfoque semánticodiscursivo posibilita identificar pasos o etapas en los textos a medida que se reconocen cambios en los patrones de recursos utilizados que los convierte en una unidad, es decir, los pasos de su estructura esquemática y luego del género (EGGINS, 1994).

\begin{tabular}{|c|c|c|c|c|}
\hline \multicolumn{2}{|c|}{ CONTEXTO } & \multicolumn{3}{|c|}{ LENGUAIE } \\
\hline \multirow{4}{*}{ 总 } & $\begin{array}{c}\text { Variable de } \\
\text { registro }\end{array}$ & $\begin{array}{c}\text { Tipo de } \\
\text { significado }\end{array}$ & $\begin{array}{c}\text { Patrones semántico- } \\
\text { discursivos }\end{array}$ & $\begin{array}{l}\text { Patrones léxico- } \\
\text { gramaticales }\end{array}$ \\
\hline & Campo & Ideacional & $\begin{array}{c}\text { IDEACIÓN } \\
\text { CONEXIÓN } \\
\text { (RELACIONES LOGICAS) }\end{array}$ & $\begin{array}{l}\text { TrANSTTVIDAD } \\
\text { RELACOONES LÓGIOO- } \\
\text { SEMÉNATCAS (TAXSS) }\end{array}$ \\
\hline & Tenor & Interpersonal & $\begin{array}{l}\text { NEgoclaóón } \\
\text { Valopación }\end{array}$ & $\begin{array}{c}\text { MOOOO } \\
\text { MODALDAD }\end{array}$ \\
\hline & Modo & Textual & $\begin{array}{l}\text { IDENTIACACIÓN } \\
\text { PERIODICIDAD }\end{array}$ & $\begin{array}{c}\text { Tema } \\
\text { Información }\end{array}$ \\
\hline
\end{tabular}

\section{Quadro 1: Relaciones entre Contexto y Lenguaje (adaptado de EGGINS; MARTIN, 2003, MARTIN; ROSE, 2007 por MOYANO, 2017)}

Esta compleja descripción contiene los principios que, a nuestro juicio, ofrece un recorte de qué enseñar cuando se pretende enseñar a leer y escribir géneros del ámbito académico o científico, como también los géneros que pasan por una trasposición didáctica y son recontextualizados para el ámbito escolar. A partir de los contenidos que corresponden a cada año de PDL, se puede pensar sin dificultades una secuenciación de géneros de menor a mayor complejidad que pueden ser enseñados en el transcurso del año lectivo en $1^{\circ}, 2^{\circ}$ y $3^{\circ}$ año de secundaria.

\section{Una opción didáctica}

Atendiendo al interrogante que ordena este artículo, hemos sentado el precedente de que existe un vacío didáctico-metodológico muy notorio en el diseño curricular de PDL vigente respecto de cómo trabajar el objeto lengua, a los fines de enseñar habilidades de lectura, escritura y comunicación.

En este sentido, desde la perspectiva adoptada aquí el problema de tener que pensar o buscar una didáctica para enseñar un objeto determinado no ocurre, dado que la LSF, justamente, ha desarrollado a partir de los años 80's una propuesta didáctica cimentada en la teorización y descripción realizada sobre el lenguaje, que inherentemente la piensa como objeto de enseñanza. De hecho, desde esta teoría se afirma que dependiendo la instrucción pedagógica que se aplique podremos, 0 bien profundizar la brecha existente entre niños expuestos a la cultura letrada desde bebés de los niños que acceden a la cultura letrada por primera vez en el sistema escolar, o bien achicar dicha brecha a través de una instrucción pensada para democratizar las habilidades de lectura y escritura, que, en definitiva, son habilidades de socialización, (ROSE \& ACEVEDO, 2007).

Por este motivo, la opción elegida en este artículo toma la propuesta didáctica de la Escuela de Sydney, que en términos generales, se puede clasificar como una didáctica de la visibilidad y la intervención (MARTIN, 1999a). Esto quiere decir que, por un lado, la visibilidad se relaciona con un alto grado de explicitud en el acto de enseñanza misma, lo que Bernstein (1990) denomina pedagogía de lo explícito, que consiste en mostrar en el diálogo con los estudiantes qué se está aprendiendo, para qué y por qué al tiempo que se da un contenido " $x$ ". Por otro lado, la intervención se relaciona con el rol docente en cada etapa de este trabajo explícito con los alumnos, dado que durante el desarrollo de las habilidades de lectura y escritura necesitarán del apoyo experto mientras realizan las tareas previstas para lograr un aprendizaje de un género en concreto y poder insertarse en una comunidad específica. En el modelo adoptado aquí, entonces, el rol docente activo es fundamental, y rompe tanto con la visión de un rol docente colocado en el centro de la escena y que sólo delega al alumno lo que este puede hacer, así como también se separa 
de una visión del docente como mero propiciador de contextos de aprendizaje (MOYANO, 2007; 2013). En contraposición con esto, lo que aquí se propone es concebir al docente como una guía experta que orientará activamente a los estudiantes a través de un andamiaje, por lo que no sólo harán lo que puedan en un momento dado, sino que mediante la guía docente y el acompañamiento de los otros pares, los alumnos llegarán a un conocimiento que estaba antes por encima de su alcance (WOOD, BRUNER \& ROSS, 1976). Posteriormente, Rose; Martin (2012) ya no hablarán de andamiaje para referirse a este proceso, sino que lo piensan como el desarrollo de una interacción en el marco de una experiencia compartida, puesto que el énfasis ya no está situado en el apoyo sino en el intercambio mutuo, que deviene luego en apoyo. Al mismo tiempo, el modelaje será otra herramienta pedagógica sumamente importante para el aprendizaje en esta propuesta (MOYANO, 2007; 2013).

En definitiva los docentes deben promover la participación de los alumnos y estimular discusiones acerca de los aspectos que competen al género y al texto en su contexto, pudiendo así reflexionar sobre el registro, los recursos semántico-discursivos y léxicogramaticales que lo realizan en el marco de actividades de enseñanza-aprendizaje concretas (MARTIN, 1999b; MARTIN; ROSE, 2005; 2012; MOYANO, 2007). En todo este proceso, el docente deberá intervenir en momentos precisos guiando y delegando continuamente para propiciar que la zona de desarrollo potencial sea zona de desarrollo real (VIGOTSKY, 1988), o en otras palabras, yendo de la heteronomía a la autonomía del alumno (MOYANO, 2007). En virtud de este principio estructurador, el modelo didáctico adoptado comienza en cada etapa con un trabajo conjunto entre el docente y el curso, en el que el docente guía a los alumnos en aquellos aspectos que estos aún no dominan individualmente. Es decir, que en el trabajo conjunto el docente, modela explícitamente qué mirar, qué considerar, cómo realizar un análisis puntual mediante preguntas orientadoras que motorizan la reflexión del estudiante (MOYANO, 2007).
Otro aspecto sumamente importante para este modelo es no frustrar a los estudiantes en el camino de aprendizaje de géneros del ámbito científico en contexto escolar, por ello se debe poder brindar posibilidades a todos los estudiantes de obtener buenos resultados en cada una de las actividades que se propongan para conocer un género. Esto se logra mediante recursos didácticos específicos que permiten dar conocimiento explícito sobre los textos, y que permiten que los alumnos reciban una preparación, una suerte de 'pista', para dar una respuesta acertada respecto de lo que se le pregunte acerca de recursos del lenguaje y los significados producidos, y de esa manera contribuir para achicar la brecha entre los alumnos "exitosos" y los alumnos que siempre cargan con el fracaso escolar (MARTIN; ROSE, 2005). Precisamente, desde esta mirada, y en consonancia con lo que proponen autores como Baquero (2000), no es el alumno quien fracasa, sino que el fracaso se debe a un conjunto de variables, entre las cuales la pedagogía implementada puede contribuir. Desde esta perspectiva, si todos los estudiantes reciben instrucción explícita, ya no serán solo los niños con más cultura letrada los que conocerán esas reglas implícitas inherentes a los ámbitos ligados al conocimiento vertical (BERNSTEIN, 1990), sino que todos tendrán la oportunidad de ejercitarse en actividades sociales de mayor abstracción. Consecuentemente, además de la preparación para dar respuestas adecuadas, los alumnos necesitarán recibir una retroalimentación, evaluación y comentarios a esas intervenciones que estarán vinculadas a la identificación de recursos predominantes en el texto de que se trate (MARTIN \& ROSE, 2005; ROSE; MARTIN, 2012).

Como este modelo propone el aprendizaje de la escritura a partir de la lectura e identificación de los significados producidos, dada la concepción de lenguaje propuesta por Halliday (1993), el modelo didáctico parte de esta premisa en su estructuración. De esta forma, el modelo que consideramos está tomado de la adaptación de Moyano (2007) del modelo original de la Escuela de Sydney (MARTIN, 1999a). En la Figura 3 se observa que la propuesta de 
Moyano exige tres etapas o momentos: a) Deconstrucción de un texto como instancia de un género; b) Construcción de un texto perteneciente a ese mismo género y c) Edición del texto producido. Cabe destacar que la etapa de Construcción está mediada por una subetapa previa a la escritura del texto, que según Moyano (2007), es su Diseño. Todas las etapas del modelo están atravesadas por la reflexión acerca del campo y el género del texto. De igual manera, todas las etapas comienzan con una fase de trabajo en conjunto, seguida de un trabajo en pequeños grupos o pares y culmina con un trabajo individual, justamente respetando el andamiaje en cada caso, tal como se observa en la Figura 3:

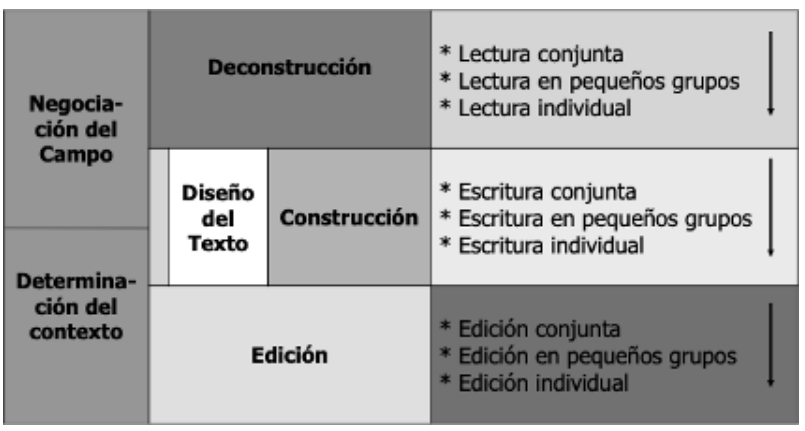

Figura 3: Propuesta didáctica (adaptado de MOYANO, 2007; 2011; 2013)

En la Deconstrucción se elige uno o dos textos del género que se quiera trabajar y que se espera los alumnos logren apropiarse. Los géneros involucrados deberán ser géneros que los estudiantes necesiten, primeramente, para comprender significados desplegados en otras asignaturas (Biología, Historia, Física, Química, etc.), así como también géneros que se precisen como antecedente para dominar géneros más complejos a medida que se avanza en el sistema educativo. En esta etapa en particular, se inicia la reflexión acerca del campo del texto en cuestión, por lo que el docente guía la interacción con los estudiantes a través de mostrar los recursos ideacionales que están construyendo significados de un campo determinado. Seguidamente, en este paso se identifica el género del texto partiendo de la actividad social llevada a cabo, el contexto en que podría circular ese texto, el destinatario, el rol asumido por el escritor, el canal utilizado (es decir, si es oral o escrito), etc.
Por otra parte, en la Deconstrucción también se identifican las partes o etapas que componen ese género, esto es, su estructura esquemática a partir de identificar los significados desplegados en cada parte, para lo cual también se requiere reconocer recursos semántico-discursivos seleccionados por el autor para construir un significado específico, al igual que sus realizaciones léxico-gramaticales, cuando sea necesario. Esta tarea se realiza, en primer lugar, de manera conjunta, siendo el docente quien modela la lectura del texto y andamia la comprensión de los estudiantes mediante explicaciones y preguntas que orienten su reflexión acerca de las características genéricas y discursivas puestas en juego en la construcción de significados del texto. De esta forma, el estudiante no solo "comprende" el texto en el sentido generalizado del término, es decir, comprende un significado " $x$ ", sino que además reconoce estrategias discursivas puntuales que podrá aplicar al momento de abordar la lectura en pequeños grupos 0 individualmente, entonces, comprende el texto porque puede reconocer los recursos lingüísticos que construyen esos significados.

En la etapa de Construcción de un texto, se respeta en general la temática propuesta por el texto modélico, aunque podría cambiar respetando el campo, y se insta a los alumnos a producir un texto del género deconstruido anteriormente. En el mejor de los casos, la construcción conjunta tiene lugar como primera instancia de escritura, funcionando no solo como modelaje del proceso de escritura, sino además de repaso de las características genéricas y recursos lingüísticos más importantes. Luego, se realiza el mismo proceso pero en pequeños grupos o pares, delegando las decisiones discursivas a los grupos de alumnos. En una última instancia, es el alumno de manera individual quien afronta la tarea de producir un texto sobre un tema poniendo en juego todo lo aprendido acerca del género y los recursos gramaticales más relevantes. Sin embargo, previo a todo este circuito de trabajo, debe haber al menos una instancia de Diseño del texto, preferentemente Diseño conjunto y Diseño en grupos, para guiar a los alumnos sobre qué contenidos incorporar en el texto, cómo 
distribuirlos en los pasos del género, y qué recursos semántico-discursivos pueden realizar esos significados oportunamente.

A diferencia de otras didácticas de la lectura y la escritura, en este marco no se corrigen ni se marcan los textos una vez que los alumnos los entregan al docente. Sino que, el docente en su rol de guía experta evalúa la calidad de los textos producidos y los devuelve sin ninguna marca, principalmente, porque consideramos que las correcciones de los docentes son ininteligibles para los estudiantes, o les generan frustración, o incluso al realizar la segunda entrega escriben textos peores que la primera versión (MOYANO, 2013). Por tal razón, aquí se propone una etapa de Edición de los textos, a la cual se le dedica tanto tiempo como al resto de las etapas, por la importancia de esta práctica en el proceso de escritura mismo.

En consecuencia, la etapa de Edición también consta de estas tres dinámicas de trabajo, Edición conjunta, en grupos o pares e individual del propio texto. En la Edición conjunta, el docente toma un texto que logre el género y otro que necesite de mucha edición para modelar, nuevamente, qué editar, cómo editar y cómo mejorar los textos en función de los significados construidos, en función del género en particular, y en función de los recursos semánticodiscursivos y léxico-gramaticales más adecuados para dicho significado y propósito social del texto. En los pasos posteriores, el docente realiza el andamiaje delegando la Edición a pequeños grupos y luego individualmente. Cuando todas estas etapas están cumplidas, los alumnos entregan la última versión del texto y recién allí reciben una calificación, según los parámetros considerados en una rúbrica, de manera que todos, alumnos y docentes, sepan los criterios de evaluación. En este sentido, el objetivo de revisar y editar los textos no tiene que ver con juzgar la destreza de sus autores, sino, por un lado, demostrar que la modificación continua es una parte natural del proceso de escritura, y por el otro, observar si las características enseñadas en la deconstrucción están presentes en el texto elaborado. Así, la reflexión acerca del género, la actividad social desarrollada, el registro y el lenguaje se repite cíclicamente en cada etapa de la propuesta didáctica.

\section{La enseñanza del género “explicación} secuencial"

Dentro de la taxonomía de géneros considerada (VEEL, 1997; MARTIN; ROSE, 2008; MOYANO, 2013), se establece que este género pertenece al conjunto que depende de una organización temporal relacionada con una serie de consecuencias simples. Por lo tanto, se trata de un género que mira hechos fácticos y busca, efectivamente, explicarlos. Asimismo, el propósito social de la Explicación Secuencial es explicar cómo ocurre un evento. Generalmente, los eventos coinciden con secuencias observables de actividades que tienen lugar con regularidad, por ejemplo la cadena de eventos que tiene lugar en el ciclo del agua. En dicha taxonomía, también se adjudica a este género una estructura esquemática cuyos pasos son: Fenómeno ^ Secuencia explicativa (presentada en fases). En cuanto a los recursos lingüísticos predominantes, se cuentan la organización causal de los eventos, que puede estar construida tanto por conjunciones como por metáforas gramaticales (MARTIN; ROSE, 2007). Se destaca, además, el uso de procesos materiales y participantes específicos o genéricos no humanos.

En el marco de las prácticas docentes del Profesorado en Lengua y Literatura de la Universidad Nacional de General Sarmiento, tuvo lugar la experiencia de planificar y efectuar la enseñanza de este género en un 3er año de PDL de una escuela pública de la localidad de San Miguel, Pcia. de Bs. As, en un período de cuatro clases de 2 hs. En base a la tipología presentada anteriormente, se seleccionó el Texto 1 para utilizar en la fase de deconstrucción conjunta para una primera aproximación al género.

\section{Origen y ubicación del sistema solar}

El universo tiene unos 15.000 millones de años de antigüedad. El sistema solar, por su parte, comenzó a formarse hace algo más de 4.500 millones de años.

Una gran nube de gas (principalmente hidrógeno y helio) y polvo interestelar comenzó a rotar y a contraerse, por efecto de la gravedad, hacia un punto central. 
En ese punto, la densidad y la temperatura aumentaron y surgió una protoestrella, que luego formó al Sol. El resto de la nube en rotación se aplanó y formó un disco.

Luego, el viento solar expulsó los materiales más livianos (gases) hacia la zona exterior del disco. El material pesado (metales y silicatos) se mantuvo más cercano al Sol.

En diferentes puntos del disco la materia comenzó a agregarse y formó anillos de materia. En los anillos cercanos al Sol el material "pesado" comenzó a chocar y formó los protoplanetas, de hasta 1000 kilómetros de diámetro. En las zonas alejadas ocurrió algo similar con los materiales más livianos, gases y hielos.

Los planetas terminaron de formarse por el choque de los protoplanetas. En cierto momento, debido a su cantidad de materia, los planetas lograron una gravedad suficiente para acaparar toda la materia de sus cercanías y limpiar sus órbitas, algo que los distingue de los planetas enanos.

Texto 1: Manual de Biología de $2^{\circ}$. Ed. Puerto de Palos. Buenos Aires, 2015.

Siguiendo la metodología sugerida, en la Deconstrucción conjunta se leyó y analizó el texto en interacción con los estudiantes. El docente fue quien guió las reflexiones de los estudiantes en la diálogo y quien fue asegurando las conclusiones sobre el género a las que arribaron juntos. Es sumamente importante cerciorarse de que todos los estudiantes participen en esta primera parte del trabajo con el género, para que adquieran seguridad y conozcan las herramientas de análisis de la etapa posterior, de modo que ni la forma de trabajo ni los criterios sean implícitos en las clases siguientes. Durante ese diálogo, y con la intención de propiciar momentos de preparación, foco, retroalimentación, evaluación y comentarios (ROSE; MARTIN, 2012) el docente fue orientando el análisis desde lo más general a lo más abstracto, empezando por el propósito social, el campo del texto, la estructura y los recursos semántico-discursivos y léxicogramaticales relevantes. Una vez completo este proceso, es aconsejable brindar actividades prácticas que ayuden a sostener los aprendizajes construidos durante el diálogo, ya que esta modalidad requiere de mucha concentración por parte del alumno. En este sentido, pensar actividades que propongan otra dinámica de trabajo y que reafirmen los conceptos es muy beneficioso didácticamente. En este caso, se ofrecieron dos actividades: 1) solicitar la representación en dos esquemas de las relaciones de hiponimia e hiperonimia halladas en el texto; 2) ordenar en un esquema cíclico los eventos naturales que, según el texto, dieron origen al sistema solar. Así, la consigna 1), por ejemplo, obligó a los alumnos a reconocer qué entidad es una clase de otra entidad. La consigna 2) exigió el reconocimiento de los eventos que posibilitaron la creación del sistema solar y las relaciones causales entre ellos.

En la clase de Construcción, los alumnos, primeramente, realizaron un repaso de lo visto hasta allí sobre el género Explicación Secuencial referenciando el texto leído. Luego de ese primer diálogo inicial, los alumnos recibieron información para poder construir un nuevo ejemplar del género. Esta información debe suponer alguna complejidad que evite la inercia de copiar párrafos u oraciones completas, por ello se sugiere que la información esté en formato de cuadros, líneas de tiempo, infografías, con los verbos sin conjugar o nominalizados, con el objetivo de que el alumno haga el trabajo de transformar esa información utilizando recursos del lenguaje y convirtiéndolo en un texto de un género particular. Para ello se proporcionó el Cuadro 2, con el cual realizaron el diseño conjunto junto con la docente y luego la construcción en parejas pedagógicas.

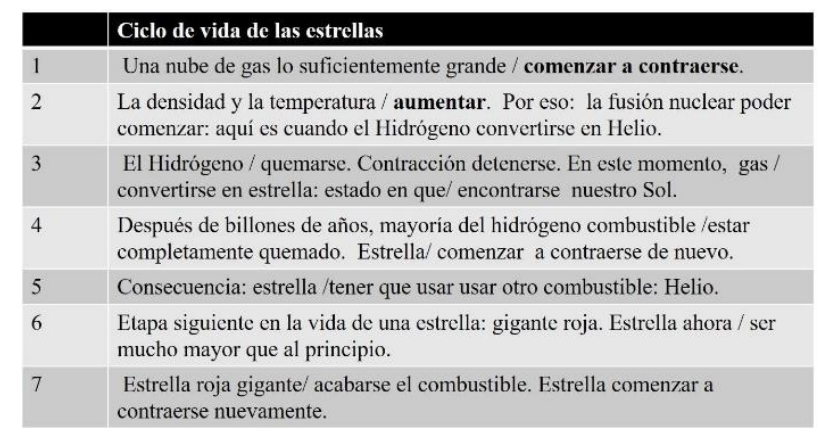

\section{Cuadro 2: Fragmento del cuadro de información para la construcción conjunta. (Elaboración propia).}

Una vez que los alumnos recibieron la información, la docente efectuó las explicaciones y preguntas necesarias para controlar que el campo del texto se comprendiera. En el Diseño conjunto la 
docente hizo hincapié, nuevamente mediante momentos de preparación, retroalimentación y evaluación, en la estructura que debía tener el texto a construir, la distribución de la información, los momentos de anticipación, de síntesis de información, es decir, todo aquello relacionado al patrón semánticodiscursivo de la periodicidad. De acuerdo con Rose; Martin (2007), en los textos existen momentos de "picos" de prominencia textual y "picos" de depresión de dicha prominencia. En los picos de prominencia discursivos se anticipa lo que se va a desarrollar o sintetiza lo ya desarrollado. De ese modo, podemos identificar los hiperTema (una oración o un grupo de oraciones que predicen una interacción particular entre cadenas léxicas y la selección del Tema en las oraciones siguientes); macroTema (un grupo de oraciones, posiblemente un párrafo, que predice un conjunto de hiperTemas); hiperNuevo (acumulación de información nueva en una oración final o un párrafo, su función es sintetizar información); y macroNuevo (acumulación de la información nueva de un texto), (MARTIN; ROSE, 2007).

En síntesis, la docente guió la planificación conjunta tanto de lo que refiere al estrato semánticodiscursivo como al estrato léxico-gramatical. En este cuadro en particular los alumnos debieron reflexionar sobre la forma verbal, es decir, qué tiempo y persona asignarle a los verbos presentados en infinitivo, y en el orden correcto de la cadena de eventos que hicieron surgir las estrellas. Después de esa primera parte, las parejas se dedicaron a construir los textos realizando consultas al docente cuando fuera necesario. Cuando el texto estuvo terminado, se lo entregaron al docente para que los revisara.

En la etapa de Edición los alumnos recibieron su texto sin marcas. La docente, por su parte, había revisado todos los textos y seleccionado uno que no necesitara de mucha edición y otro que necesitara de un trabajo más profundo, como se anticipó en el apartado anterior. Los alumnos, entonces, recibieron ambos textos y de manera conjunta con el docente los editaron, recuperando las nociones principales: propósito del género, campo construido, estructura esquemática, recursos semántico-discursivos y léxico- gramaticales, como también cuestiones de normativa, puntuación y acentuación. Una vez que la docente mostró cómo efectivizar, paso por paso, la Edición, delegó esta tarea a las parejas con su propio texto para luego entregarlo y recibir una calificación numérica.

Finalmente, los alumnos volvieron a realizar el proceso de Construcción pero individualmente en base a una infografía sobre los agujeros negros (Figura 4). La docente volvió a sostener el diseño conjunto y delega la escritura a cada estudiante. Posteriormente, se realizó la edición individual del texto de un compañero, con el que luego debían discutir sus sugerencias de cambio y entregar la versión final.

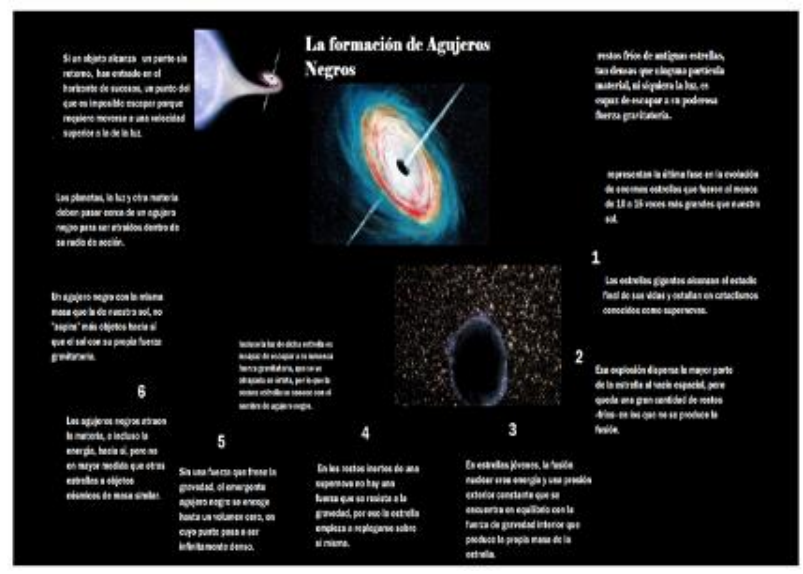

Figura 4: Infografía para la construcción individual. (Elaboración propia).

\section{Resultados}

El objetivo principal de la aplicación de esta secuencia didáctica fue enseñar ciertos recursos lingüísticos al servicio de las características genéricas de la Explicación Secuencial, que es un género muy recurrente en los años de la secundaria básica en disciplinas de las ciencias naturales. Los resultados de las producciones textuales de los estudiantes fueron en todos los casos muy positivas, se lograron avances en habilidades de escritura en todos los estudiantes. Los que presentaban mayores problemas en competencias discursivas lograron producir textos acordes al género solicitado y adecuados en la utilización de recursos del lenguaje. A su vez, los alumnos que ya presentaban buenas condiciones discursivas también mejoraron en sus habilidades de 
escritura, dado que pudieron profundizar en el manejo de recursos del lenguaje del estrato semánticodiscursivo y léxico-gramatical, que con otro tipo de instrucción, tal vez, resulta más difícil apropiarse. Sostenemos que la instrucción explícita y la facilitación del metalenguaje pertinente posibilitaron que todos los alumnos lograran alcanzar los objetivos pedagógicos, que todos ellos produjeran nuevos aprendizajes, y que todos manejaran la misma información en el mismo momento y para los mismos fines.

Además, se logró la participación de los estudiantes que mantenían una relación de baja intensidad con los contenidos de la asignatura PDL, e incluso resultados positivos en sus producciones textuales. A continuación, presentaremos el Texto 2, correspondiente a la Explicación Secuencial en su primera versión, y el Texto 3 , correspondiente a la versión editada, para observar el avance que da cuenta del resultado del proceso desarrollado.

\section{La formación de los agujeros negros}

Los agujeros negros son restos fríos de antiguas estrellas tan densas que ninguna partícula material (ni siquiera la luz) es capaz de escapar de su poderoza fuerza gravitatoria.

La formación de los agujeros negros comienza, cuando las estrellas gigantes alcanzaron el estadio final de sus vidas, y estallaron en cataclismos como supernovas.

En segundo lugar, esa exploción dispersó la mayor parte de la estrella al vacío espacial, pero dejando una gran cantidad de restos fríos en los que se produjo la fusión. Por esta razón en las estrellas jóvenes, la fución nuclear creó una energía y una preción exterior constante que se encontró en equilibrio con la fuerza de gravedad interior que produjo la propia masa de la estrella.

Por lo que, los restos inertes de una supernova, no huvo fuerza que se resista a la gravedad, a causa de eso, la estrella empezó a replegarse sobre sí misma. Por lo que, sin una fuerza que frene la gravedad, el emergente agujero negro se encojió hasta un volumen cero, en cuyo punto pasó a ser infinitamente denso.

Texto 2: Versión 1 de la explicación secuencial.

\section{La formación de agujeros negros}

Los agujeros negros representan restos fríos de antiguas estrellas tan densas, que ninguna partícula material, ni siquiera la luz, es capaz de escapar a su poderosa fuerza gravitatoria.

Su formación comenzó cuando las estrellas gigantes alcanzaron el estadio final de sus vidas y estallaron en cataclismos conocidos como supernovas. Debido a esta explosión, la mayor parte de la estrella se dispersó al vacío espacial, sin embargo, quedó una gran cantidad de restos fríos en los que no se produjo la fusión.

En estrellas jóvenes, la fusión nuclear creó una energía y una presión exterior constante que se encontró en equilibrio con la fuerza de gravedad interior, que, a su vez, produjo la propia masa de la estrella. Como en los restos inertes de la supernova no hubo una fuerza que se resistiera a la gravedad, la estrella empezó a replegarse sobre sí misma. así, sin una fuerza que frenara la gravedad, el emergente agujero negro se encogió hasta un volumen cero, en cuyo punto pasó a ser infinitamente denso.

Finalmente, los agujeros negros atrajeron la materia e incluso la energía hacia sí, pero no en mayor medida que otras estrellas u objetos cósmicos de masa similar. Por lo tanto, los planetas, la luz y otras materias deben pasar cerca del agujero negro para ser atraidas dentro de su radio de acción.

Texto 3: Versión 2 de la explicación secuencial

Como se puede observar, la diferencia entre la versión 1 y la versión 2 es realmente notable en distintos niveles. Primeramente, es preciso señalar que esta ya era la segunda Explicación Secuencial que elaboraban, sólo que en este caso la Construcción y Edición fueron individuales. En la primera versión, las oraciones tienden a ser muy densas para ser leídas, la información no está desplegada de manera tan clara y algunos conectores son imprecisos en la posición o en la oración que son colocados: "Por esta razón en las estrellas jóvenes, la fución nuclear creó una energía y una preción exterior constante que se encontró en equilibrio con la fuerza de gravedad interior que produjo la propia masa de la estrella" (Texto 2). En este ejemplo se introducen varios pasos del proceso de formación de agujeros negros al mismo tiempo, sin dar cuenta de las relaciones causales que se generan entre los distintos eventos involucrados. Tampoco hay consistencia en la identificación del Tema (MOYANO, 2016), por lo que se dificulta captar el método de desarrollo, es decir, el aspecto del campo sobre el cual 
se llama la atención del lector: "Por lo que, los restos inertes de una supernova, no huvo fuerza que se resista a la gravedad, a causa de eso, la estrella empezó a replegarse sobre sí misma" (Texto 2). Finalmente, hay algunos detalles de ortografía y prolijidad que en la segunda versión tienden a mejorar notoriamente: "(..) su poderoza fuerza gravitatoria." (Texto 2).

Por su parte, en la versión 2 del texto el alumno mejora el manejo del flujo de información, los párrafos componen unidades claras de sentido y las oraciones que los componen están completas y son precisas en la construcción del significado en cuestión. La utilización de conectores para vincular ideas es mucho más ordenada y específica, los referentes de las cláusulas son fácilmente reconocibles, y no se dificulta identificar la estructura del género ni la progresión del fenómeno natural explicado:

\begin{abstract}
"Su formación comenzó cuando las estrellas gigantes alcanzaron el estadio final de sus vidas y estallaron en cataclismos conocidos como supernovas. Debido a esta explosión, la mayor parte de la estrella se dispersó al vacío espacial, sin embargo, quedó una gran cantidad de restos fríos en los que no se produjo la fusión." (Texto 3).
\end{abstract}

En este ejemplo se observa que se recupera correctamente el Tema textual cada vez que es necesario (agujeros negros, estrellas, restos fríos), la sucesión de eventos que dan lugar a la formación de agujeros negros es clara y se evidencia discursivamente cómo cada evento permite el siguiente en el proceso explicado. Asimismo, el léxico es mucho más técnico que en el Texto 2 (sobre todo en la elección de procesos materiales: estallar, producir, alcanzar). Los conectores son colocados en posiciones que contribuyen a construir el significado global, y son seleccionados en función de la relación lógica que se quiere expresar. Además, el autor logra dar un desarrollo y una explicación completa del fenómeno natural particular que recorre desde el inicio de los eventos que componen su formación hasta el final: "Finalmente, los agujeros negros atrajeron la materia e incluso la energía hacia sí, pero no en mayor medida que otras estrellas u objetos cósmicos de masa similar" (Texto 3). En definitiva, mejora ampliamente, sobre todo, la utilización de recursos semánticodiscursivos y léxico-gramaticales, dado que en la versión 1 ya estaba logrado el género, el campo del texto y su propósito social.

\section{Consideraciones finales}

Hasta aquí, hemos presentado los principios teóricos fundamentales de la LSF que vertebran las ventajas de elegir una opción teórica, que trabaja la enseñanza del lenguaje a partir de la reflexión sobre el género, el registro, y sus realizaciones lingüísticas. Seguidamente, hemos desarrollado la propuesta didáctica que parte de un diseño pedagógico elaborado por la Escuela de Sydney, y adaptado por Moyano (2007). Luego, hemos expuesto detalladamente la aplicación de dicha propuesta en la enseñanza del género explicación secuencial en un $3^{\circ}$ año de escuela media en la localidad de San Miguel, Bs. As., Argentina. Finalmente, hemos considerado sus resultados a partir del relevo de un ejemplo particular como muestra representativa del resto del curso con el que se trabajó.

A modo de conclusión, nos interesa destacar que el mayor desafío enfrentado durante la aplicación de la propuesta y secuencia didáctica elaborada tuvo que ver con la falta de tiempo para trabajar mayor metalenguaje y sostener de mejor manera los diálogos de análisis textual de forma conjunta con los alumnos. Al contrario de lo que suponen otros enfoques o teorías, el metalenguaje hace la diferencia si tiene inmediatamente sentido respecto del significado construido en el texto, y deja de ser mero repositorio de etiquetas que no permiten, a priori, apropiarse de los recursos del lenguaje o conocer su verdadera naturaleza en el discurso. Por otra parte, las resistencias que se encontraron en la implementación de la secuencia no fueron por parte de los estudiantes, quienes rápidamente se amoldaron a la metodología de trabajo, sino de la propia docente que, como responsable del curso oficiaba como co-formadora de la residente. Esto probablemente se debió al desconocimiento de la propuesta, de manera que no 
pudo participar ni aportar demasiado al proceso involucrado. En el futuro, se espera poder seguir ajustando lo que se vincula con las interacciones con los estudiantes en los momentos de Deconstrucción y Edición conjunta, esto es, poder desarrollar pautas específicas para trabajar el contexto y el campo de la manera más eficiente posible en cualquier género científico.

\section{Referências}

BAQUERO, Ricardo. Lo habitual del fracaso y el fracaso de lo habitual. En AVEDAÑO, Fernando; BOGGINO, Norberto (comps.), La escuela por dentro y el aprendizaje escolar. Rosario: Homo Sapiens, 2000.

BERNSTEIN, Basil. Class, codes and control. Vol. 4: The structuring of pedagogic discourse. London: Routledge, 1990.

CORTES, Marina. Los textos. Marcos teóricos y prácticas de enseñanza. En

ALVARADO, Maite (coord.) Entre líneas. Teorías y enfoques en la enseñanza de laescritura, la gramática y la literatura. Buenos Aires: Manantial, 2001. P. 113144.

\section{DIRECCIÓN GENERAL DE CULTURA Y} EDUCACIÓN. Diseño curricular para la educación secundaria. Buenos Aires, 2006. 420 p.

EGGINS, Suzanne. An introduction to systemic functional linguistics. London: Cassell, 1994.

EGGINS, SUZANNE; MARTIN, James. El contexto como género: una perspectiva lingüístico-funcional. Revista Signos, v. 36, n.54, p. 185-205. 2003.

HALLIDAY, Michael. Text Semantics and Clause Grammar: How is a Text like a Clause? En WEBSTER, Jonathan. (Ed.), On Grammar. Collected Works of M. A. K. Halliday, v. 1, p. 219-260. London: Continuum, 1981 [2002].

HALLIDAY, Michael. The Notion of "Context" in Language Education. En WEBSTER, Jonathan (Ed.), Language in Education. Collected Works of M. A. K. Halliday, v. 9, p. 269-290. London: Continuum, 1991 [2007].

HALLIDAY, Michael. The Act of Meaning. En WEBSTER, Jonathan (Ed.), On Language and Linguistics. Collected Works of M. A. K. Halliday, London: Continuum, v. 3, p. 375-389. 1992 [2003].

HALLIDAY, Michael. Towards a Language-based theory of learning. Linguistics and Education, v.5, n. 2, p. 93-116. 1993.
MARTIN, James. Process and text: two aspects of human semiosis. En BENSON, James; GREAVES, William (Eds.). Systemic Perspectives on Discourse: selected theoretical papers from the 9th, International system workshop. Norwood, N.J.: Ablex, 1985. P. 248-274.

MARTIN, James. English Text: System and Structure. Amsterdam: Benjamins, 1992.

MARTIN, James. Analyzing genre: functional parameters. En CHRISTIE, Frances; MARTIN, James (Eds). Genre and Institutions: Social processes in the work place and School. London: Continuum, 1997. P. 3-39.

MARTIN, James. Modelling context: a crooked path of progress in contextual linguistics (Sydney SFL). En GHADESSY, Mohsen (Ed). Text and Context in Functional Linguistics. Amsterdam: Benjamins (CILT Series IV),1999a. P. 25-61.

MARTIN, James. Mentoring semogenesis: 'genrebased' literacy pedagogy. En CHRISTIE, Frances (Ed). Pedagogy and the Shaping of Consciousness: linguistic and social processes. London: Cassell (Open Linguistics Series), 1999b. P. 123-155.

MARTIN, James; ROSE, David. Designing Literacy Pedagogy: scaffolding asymmetries. En WEBSTER, Jonathan, MATTHIESSEN, Christian; HASSAN, Ruqaia (Eds.). Continuing Discourse in Language. London: Continuum, 2005. P. 251-280.

MARTIN, James; ROSE, David. Working with Discourse: Meaning beyond the Clause. 2nd Ed. London: Continuum, 2007.

MARTIN, James \& ROSE, David. Genre Relations. Mapping culture. London: Equinox, 2008.

MOYANO, Estela. Enseñanza de Habilidades Discursivas en Español en Contexto Pre-universitario: Una Aproximación desde la LSF. Revista Signos, vol. 40, n. 65, p. 573-608, 2007.

MOYANO, Estela. Deconstrucción y Edición Conjuntas en la enseñanza de la escritura: La reflexión sobre género y discurso en la formación académica y profesional. Anais VI Simpósio Internacional de Estudios de Géneros Textuais (VI SIGET), Lagoa Nova, Natal: Universidade Federal do Rio Grande do Norte, p. s/n, 2011 a.

MOYANO, Estela. Una didáctica de las ciencias basada en los géneros textuales: acceso a las disciplinas a través de la apropiación de su discurso. En MOYANO, Estela (Coord.), Aprender ciencias y humanidades: una cuestión de lectura y escritura. Aportes para la construcción de un programa de 
inclusión social a través de la educación lingüística: Argentina. Los Polvorines: UNGS, 2013a. 109-155 p.

MOYANO, Estela. Diseño e implementación de programas de Lectura y escritura en el nivel universitario: Principios y estrategias. Lenguas modernas, Universidad de Chile, v. 50, p. 47-72, 2017.

OTAÑI, Laiza; GASPAR, M. del Pilar. Sobre la gramática, en Maite Alvarado (coord.) Entre líneas.

Teorías y enfoques en la enseñanza de la escritura, la gramática y la literatura. Buenos Aires: Manantial, 2001. P. 75-112.

ROSE, David; ACEVEDO, Claire. Reading and writing to learn in the middle years of schooling. NSW: Primary English Teaching Association (PETA), 2007.

ROSE, David; MARTIN, James. Learning to Write, Reading to Learn: Genre, knowledge and pedagogy in the Sydney School. London: Equinox, 2012.

VEEL, Robert. Learning how to mean -scientiffically speaking. Apprenticeship into scientific discourse in the secondary school. En CHRISTIE, Frances; MARTIN, James (Eds.). Genre and institutions. Social proceses in the workplace and school. London: Contiuum, 1997. P. 161-195.

VIGOTSKY, Lev. El desarrollo de los procesos psicológicos superiores. Méxi-co: Crítica Grijalbo, 1988. P. 39-56, 87-94, 123-140.

WOOD, David; BRUNER, Jerome; ROSS, Gail. The Role of Tutoring in Problem Solving. Journal of Child Psychology and Psychiatry, vol.17, p. 89-100, 1976.

\section{COMO CITAR ESSE ARTIGO}

NIETO, Florencia Gimena. La enseñanza del género explicación secuencial en el aula de Prácticas del Lenguaje. Signo, Santa Cruz do Sul, v. 46, n. 86, may 2021. ISSN 1982-2014. Disponível em: <https://online.unisc.br/seer/index.php/signo/article/view/1 6065>.doi:https://doi.org/10.17058/signo.v46i86.16065. 